\title{
Evidence for the Hanle effect in molecular lines
}

\author{
S. V. Berdyugina ${ }^{1,2}$ and D. M. Fluri ${ }^{1}$ \\ 1 Institute of Astronomy, ETH Zentrum, 8092 Zurich, Switzerland \\ e-mail: fluri@astro.phys.ethz.ch \\ 2 Astronomy Division, PO Box 3000, 90014 University of Oulu, Finland
}

Received 6 October 2003 / Accepted 15 December 2003

\begin{abstract}
In many wavelength regions molecular lines dominate the second solar spectrum that results from coherent scattering. Scattering polarization is modified by magnetic fields via the Hanle effect. This allows us to explore the magnetic field regime with weak field strengths and mixed polarities, which is not seen with the Zeeman effect and thus contains complementary information. Molecular lines are particularly well suited to diagnose such turbulent fields because they exhibit a broad range of magnetic sensitivities within narrow spectral regions. Thus, it is possible to employ the technique of the differential Hanle effect, i.e. to obtain field strengths by observing polarization ratios in various lines. We have identified one $\mathrm{R}$ - and one P-triplet of $\mathrm{C}_{2}$ at $5140 \AA$ and $5141 \AA$, respectively, that satisfy all conditions to be used in the differential Hanle effect. Based on these lines we have developed a model that can diagnose turbulent magnetic fields using the Hanle effect. The tool is sensitive over a broad range of magnetic field strengths from a few Gauss up to several hundred Gauss. This tool has allowed us to find a significant Hanle depolarization of $\mathrm{C}_{2}$ lines in quiet Sun observations, which corresponds to a magnetic field strength of $15 \pm 3 \mathrm{G}$.
\end{abstract}

Key words. line: formation - molecular processes - polarization - scattering - Sun: atmosphere - Sun: magnetic fields

\section{Introduction}

Various phenomena of solar activity are essentially related to the magnetic field. Because of its interaction with the convective flows, the magnetic flux emerging at the solar surface is highly inhomogeneous and appears on a wide spectrum of spatial and temporal scales. The major part of the magnetic flux is arranged into large concentrations of a strong (above $1 \mathrm{kG}$ ), mainly vertical field, such as active regions, sunspots, the network and intranetwork. The field in these structures is predominantly of one polarity within a spatial resolution element. Outside active regions and sunspots the filling factor of such fields is however of about $1 \%$ of the photospheric volume. The rest is occupied by unconcentrated, mainly weak magnetic fields of mixed polarity, which is tangled up by the convective and turbulent motions. Such fields are currently spatially unresolved and may not be resolved in principle if single-polarity elements are much smaller than the photon mean free path.

In contrast to spatially resolved magnetic fields with clear separation of the opposite polarities, which are detected with the Zeeman effect, weak mixed-polarity turbulent fields are not easily visible. They are however found to influence scattering polarization in spectral lines via the Hanle effect, which at the solar limb manifests itself by decreasing the polarization degree and rotating the plane of polarization (Stenflo et al. 1998; Stenflo 2001). Since opposite polarity fields contribute with the

Send offprint requests to: S. V. Berdyugina, e-mail: sveta@astro.phys.ethz.ch same sign to Hanle depolarization, scattering polarization is a unique source of information on unresolved mixed-polarity fields.

Coherent scattering on the Sun produces a wealth of linearly polarized features in the Stokes $Q / I$ spectrum, which have been referred to as the second solar spectrum (Ivanov 1991; Gandorfer 2000). It is rich in various atomic contributions and in some spectral regions dominated by prominent polarization in molecular lines. Scattering polarization of strong atomic lines, such as $\mathrm{Na} \mathrm{I} \mathrm{D}_{2}$ and $\mathrm{Ca}$ I $4227 \AA$, exhibits large spatial fluctuations suggesting the presence of highly inhomogeneous fields in the upper solar photosphere (e.g. Stenflo et al. 2002). Molecular lines, such as of $\mathrm{MgH}$ and $\mathrm{C}_{2}$, on the contrary, apparently show no spatial variations. This has been interpreted as molecular lines not being sensitive to the Hanle effect, or by magnetic fields being more homogeneous in the layers where $\mathrm{MgH}$ and $\mathrm{C}_{2}$ lines are formed or, as proposed by Trujillo Bueno (2003c), by contributions to the emergent scattering polarization in molecular lines coming mainly from the upflowing cell centers that contain the weakest magnetic fields.

As was shown by Berdyugina et al. (2002), the polarization amplitudes and sensitivities of molecular lines to the Hanle effect significantly vary with the total angular momentum number $J$. Molecular transitions with smaller $J$ numbers are more magnetically sensitive but show smaller polarization amplitudes, while those with larger $J$ show strong scattering polarization but are rather insensitive to magnetic fields. Unfortunately, only lines with strong polarization and, hence, 
low magnetic sensitivity were used to search for spatial variations. It is not surprising, therefore, that no significant fluctuations were found (Trujillo Bueno 2003c; Faurobert \& Arnaud 2003).

A broad range of magnetic sensitivities of molecular lines observed within a narrow spectral region offers a unique opportunity to use the differential Hanle effect and develop model-independent diagnostics of spatially unresolved magnetic fields. This has raised considerable interest in the field lately. Faurobert \& Arnaud (2003) have successfully modeled the center-to-limb variation of $\mathrm{C}_{2}$ and $\mathrm{MgH}$ molecules and employed their model to infer empirically molecular data. Trujillo Bueno (2003c) has studied the sensitivity of molecular lines to the Hanle effect and has discussed the apparent spatial invariance of molecular lines.

However, until recently the Hanle effect has not been detected in molecules. We presented the first evidence for the Hanle effect in molecular lines based on preliminary analysis of $\mathrm{C}_{2}$ lines (Fluri \& Berdyugina 2003). In the second solar spectrum (Gandorfer 2000) we found $\mathrm{C}_{2}$ lines significantly affected by the Hanle effect. We concluded that these $C_{2}$ lines represent a sensitive diagnostic tool to measure weak magnetic fields in the solar photosphere. Faurobert \& Arnaud (2003) have analyzed observations of other $\mathrm{C}_{2}$ lines, which are consistent with the presence of weak magnetic fields. However, because those lines have a lower Hanle sensitivity they are useful to diagnose stronger magnetic fields, but not so reliable for weak fields.

In the present paper we analyze scattering polarization and the Hanle effect in molecular lines with a simple model and search for lines with different magnetic sensitivity. In particular, we employ the technique of the differential Hanle effect as introduced by Stenflo et al. (1998) in general and further discussed by Trujillo Bueno (2003c) for molecular lines. We apply our model to the $\mathrm{C}_{2}$ lines that are significantly affected by the Hanle effect and develop a new diagnostic tool for studying turbulent magnetic fields.

\section{Model}

The observed polarization in the second solar spectrum contains contributions from the continuum polarization, depolarization of the continuum polarization in lines and line polarization. The polarization in the continuum is formed by Rayleigh and Thomson scattering. Absorption and isotropic scattering in lines reduce the anisotropy of the radiation field and destroy polarization in the continuum forming a depolarized profile in the continuum polarization. Transitions with an intrinsic polarizability $W_{2} \neq 0$ polarize the radiation field in coherent scattering processes. This line polarization is in turn reduced by elastic collisions and can be modified by magnetic fields via the Hanle effect. As shown by Fluri \& Stenflo (2003) the remaining line polarization is added to the depolarized profile. For very weak lines the depolarization of the continuum can be neglected. Then the line polarization is added to the continuum polarization. In this paper we neglect depolarization of the continuum and take the theoretical value of the continuum polarization as computed by Fluri \& Stenflo (1999).
Here we introduce a simple model to describe polarization in weak spectral lines, which is appropriate for both atomic and molecular lines. Let us consider a molecular electronic dipole transition between an upper level described with the electronic, vibrational and rotational quantum numbers $\Lambda^{\prime}, v^{\prime}$, and $J^{\prime}$ and a lower level with the numbers $\Lambda^{\prime \prime}, v^{\prime \prime}$, and $J^{\prime \prime}$. Berdyugina et al. (2002) concluded that the scattering polarization amplitudes in molecular lines depend on three factors: on the opacity factor $K_{\Lambda^{\prime \prime} v^{\prime \prime} J^{\prime \prime}, \Lambda^{\prime} v^{\prime} J^{\prime}}$, the Landé factor $g_{\mathrm{L}}$, and on the intrinsic polarizability $W_{2}$. To keep the notation short, we will further indicate only on which quantum numbers a given variable depends, for example, $K_{\Lambda v J}$. Note that Berdyugina et al. (2002) used a different notation $I_{J^{\prime \prime} J^{\prime}}$ for the same factor.

To obtain the degree of line polarization $Q / I$, we have to solve in general the radiative transfer problem and compute the Stokes parameters $Q$ and $I$ by integrating their source functions along the line of sight. For weak lines the source functions are nearly depth independent and the intensity $I$ is almost equal to the continuum intensity. Therefore, the ratio $Q / I$ is proportional to the source function of $Q$ and the optical thickness of the line. The $Q$ source function scales with the intrinsic line polarizability $W_{2}$, with the collisional depolarization factor $W_{\mathrm{c}}$ and with the Hanle depolarization factor $W_{\mathrm{H}}$. The optical thickness is proportional to the opacity factor $K_{\Lambda v J}$ and the line profile $\Phi_{\lambda}$. Other factors are not relevant to the present consideration because they drop out in the differential Hanle effect. We include them in a scaling factor $q$. Thus, we find for the degree of line polarization

$\frac{Q}{I}=q W_{\mathrm{H}} W_{\mathrm{c}} W_{2} K_{\Lambda v J} \Phi_{\lambda}$,

which is consistent with the conclusions made by Berdyugina et al. (2002). The factors in Eq. (1) are described in more detail below.

The Voigt function $\Phi_{\lambda}$ is used to account for the wavelength dependence within the line profile, which is indicated by the subscript $\lambda$. The opacity factor is given by

$K_{\Lambda v J}=g_{J^{\prime \prime}} f_{\Lambda v J} \mathcal{N}_{\max } \exp \left(-E_{J^{\prime \prime}} / k T\right)$,

where $f_{\Lambda v J}$ is the absorption oscillator strength, $g_{J^{\prime \prime}}=2 J^{\prime \prime}+1$ the statistical weight of the lower level, and $E_{J^{\prime \prime}}$ is the lower level excitation energy. The temperature $T$ and the number density of the considered molecule $\mathcal{N}_{\max }$ are taken at the height in the solar atmosphere where the line contribution function reaches the maximum. The oscillator strength is calculated according to Eq. (14).

Further, the intrinsic polarizability $W_{2}$ is determined by the same expressions for atomic and molecular transitions. For Rayleigh scattering they are given by (e.g. Stenflo 1994)

$$
\begin{aligned}
& W_{2}(\Delta J=-1)=\frac{\left(J^{\prime \prime}-1\right)\left(2 J^{\prime \prime}-3\right)}{10 J^{\prime \prime}\left(2 J^{\prime \prime}+1\right)} \\
& W_{2}(\Delta J=0)=\frac{\left(2 J^{\prime \prime}-1\right)\left(2 J^{\prime \prime}+3\right)}{10 J^{\prime \prime}\left(J^{\prime \prime}+1\right)} \\
& W_{2}(\Delta J=+1)=\frac{\left(J^{\prime \prime}+2\right)\left(2 J^{\prime \prime}+5\right)}{10\left(J^{\prime \prime}+1\right)\left(2 J^{\prime \prime}+1\right)}
\end{aligned}
$$


where $J^{\prime \prime}$ is the total angular momentum quantum number of the lower level and $\Delta J=J^{\prime}-J^{\prime \prime}$. In the molecular case the three sequences of transitions with $\Delta J=-1,0,+1$ form $\mathrm{P}, \mathrm{Q}$, and $\mathrm{R}$ rotational branches, respectively. Since the contribution to the line polarization of Raman scattering is smaller than the one of Rayleigh scattering, we neglect Raman scattering in the following.

If $W_{2}=0$ then the line polarization is zero within the current theoretical framework. For the $\mathrm{P}$ branch this is the case if $J^{\prime \prime}=1$ or $J^{\prime \prime}=1.5$, for the Q branch if $J^{\prime \prime}=0.5$, while for the R branch $W_{2}>0$ for all possible $J^{\prime \prime}$. Note that for large $J^{\prime \prime}$ numbers $W_{2}$ of transitions in the Q branch approach 0.4 , and in the $\mathrm{P}$ and $\mathrm{R}$ branches 0.1 (cf. Fig. 2 in Berdyugina et al. 2002).

In the presence of a magnetic field the emergent scattering polarization is modified by the Hanle effect (see Trujillo Bueno 2001, and references therein). For observations close to the solar limb Stokes $Q$ is always reduced except for vertical fields, which do not influence scattering polarization. For an isotropic magnetic field distribution the depolarization can be described by the factor (Stenflo 1982; Landi Degl'Innocenti \& Landi Degl'Innocenti 1988)

$W_{\mathrm{H}}=1-\frac{2}{5}\left(\frac{\Gamma_{\mathrm{H}}^{2}}{1+\Gamma_{\mathrm{H}}^{2}}+\frac{4 \Gamma_{\mathrm{H}}^{2}}{1+4 \Gamma_{\mathrm{H}}^{2}}\right)$,

with

$\Gamma_{\mathrm{H}}=0.88 \frac{g_{\mathrm{L}}^{\prime} B}{\gamma_{\mathrm{R}}+D^{(2)}}$.

The variable $g_{\mathrm{L}}^{\prime}$ denotes the Landé factor of the upper level, $B$ the magnetic field strength in Gauss. In the denominator $\gamma_{\mathrm{R}}$ is the radiative decay rate, which is discussed in Sect. 3, and $D^{(2)}$ the rate of depolarizing elastic collisions, both are for the upper level and given in units of $10^{7} \mathrm{~s}^{-1}$. Further, $D^{(2)}$ is expressed in terms of the elastic collision rate $\gamma_{\mathrm{E}}$ by

$D^{(2)}=d \gamma_{\mathrm{E}}$,

where $d=0.38$ (Stenflo 1994). In Eq. (7) the sum $\gamma_{\mathrm{R}}+D^{(2)}$ is determined by the width of the upper level and, if $D^{(2)}$ is neglected, $\gamma_{\mathrm{R}}$ is just the reciprocal of the level's lifetime, while the factor $g_{\mathrm{L}}^{\prime} B$ determines the magnetic splitting of the level. Therefore, for levels with longer lifetimes the Hanle effect shows up already for weaker fields, and it remains efficient as long as the magnetic splitting is comparable with the level's width. When the splitting is significantly larger than the level's width, the Hanle effect is saturated and $W_{\mathrm{H}}$ reaches the asymptotic value 0.2 . If $g_{\mathrm{L}}^{\prime}=0$, Hanle effect does not modify line polarization and $W_{\mathrm{H}}=1$. For molecular lines the Landé factor in general decreases as the $J$ number increases, while level lifetimes are almost constant. Therefore, the Hanle sensitivity reduces with $J$.

Elastic collisions can reduce the degree of scattering polarization. They can be taken into account by the collisional depolarization factor

$W_{\mathrm{c}}=\frac{\gamma_{\mathrm{R}}+\gamma_{\mathrm{I}}}{\gamma_{\mathrm{R}}+\gamma_{\mathrm{I}}+D^{(2)}}$, where $\gamma_{\mathrm{I}}$ is the rate of inelastic collisions. The factor $W_{\mathrm{c}}$ is thus the probability that no depolarizing elastic collision occurs during the scattering process (Domke \& Hubeny 1988; Faurobert-Scholl 1992).

The scaling factor $q$ accounts for the details of line formation, which are not included in the other factors of Eq. (1). These details contain radiative transfer effects with multiple scattering, the height of line formation, the height dependence of the anisotropy of the radiation field, branching ratios of scattering processes with respect to pure absorption, limb distance of the observation, inhomogeneities of the solar atmosphere, etc.

In the differential Hanle effect we form ratios of line polarization, thus avoiding many potential errors that could arise from further assumptions on radiative transfer or from instrumental calibration. It is essential that the model is applied to atomic or molecular lines for which the scaling factor $q$ is the same. This is true for weak lines that are formed at about the same height and have similar wavelengths. All physical parameters, which differ in those lines, are explicitly stated on the right-hand side of Eq. (1). When using the differential Hanle effect as a tool to diagnose magnetic fields, the factor $q$ drops out. On the other hand, $q$ can be determined from a sample of lines with different sensitivity to the Hanle effect or from one line with a negligible Landé factor of the upper level.

Recently, Faurobert \& Arnaud (2003) introduced a more elaborate model including explicitely many of the above stated details contained in the $q$ factor, which allows for describing also stronger lines. Their treatment, however, inevitably adds more assumptions because it depends on computing absolute values of polarization including continuum polarization. It is very important to realize that we do not have to know these details at all when using the differential Hanle effect as long as we study weak lines. In fact, we get smaller errors with our treatment since we account automatically for many complicated effects such as inhomogeneities in the solar atmosphere.

\section{Radiative lifetimes}

The average radiative lifetime of a given upper level is the inverse of the damping constant:

$\tau_{\mathrm{R}}=\frac{1}{\gamma_{\mathrm{R}}}$

The radiative damping constant of the upper level is a sum of the Einstein coefficients of the spontaneous emission for all allowed transitions to lower levels:

$\gamma_{\mathrm{R}}=\sum_{\Lambda^{\prime \prime}} \sum_{v^{\prime \prime}} \sum_{J^{\prime \prime}} A_{\Lambda v J}$

Here, to keep the notation short we denote transitions as $\Lambda v J$ instead of the explicit subscript $\Lambda^{\prime \prime} v^{\prime \prime} J^{\prime \prime}, \Lambda^{\prime} v^{\prime} J^{\prime}$. One prime always refers to the upper level and double prime to the lower level. The selection rules allow transitions between electronic states of the same multiplicity with $\Delta \Lambda=\Lambda^{\prime}-\Lambda^{\prime \prime}=-1,0,+1$ and rotational levels with $\Delta J=J^{\prime}-J^{\prime \prime}=-1,0,+1$. There are no selection rules for vibrational transitions. Therefore, the summation extends over all existing lower vibrational levels. 
The Einstein coefficient can be expressed via the absorption line oscillator strength $f_{\Lambda v J}$ :

$A_{\Lambda v J}=\frac{8 \pi^{2} e^{2}}{\lambda_{\Lambda v J}^{2} m c} \frac{g_{J^{\prime \prime}}}{g_{J^{\prime}}} f_{\Lambda v J}$,

where $g_{J^{\prime \prime}}=2 J^{\prime \prime}+1$ and $g_{J^{\prime}}=2 J^{\prime}+1$ are statistical weights of the lower and upper levels, respectively, and $\lambda_{\Lambda v J}$ is the wavelength of the transition. With $J^{\prime}=1, J^{\prime \prime}=0$ and $f_{J}=1$ we obtain the damping constant of the classical oscillator

$\gamma_{0}=\frac{1}{\tau_{0}}=\frac{1}{3} \frac{8 \pi^{2} e^{2}}{\lambda_{J}^{2} m c}$.

Since the numbers $\Lambda$ and $v$ are not defined for the classical oscillator we skip them in the notation of the wavelength.

The molecular line oscillator strength is expressed via the band oscillator strength $f_{\Lambda v}$, the wavelength of the vibrational transition $\lambda_{\Lambda v}$, and the theoretical line strength $S_{\Lambda J}$ :

$f_{\Lambda v J}=f_{\Lambda v} \frac{\lambda_{\Lambda v}}{\lambda_{\Lambda v J}} \frac{S_{\Lambda J}}{g_{J^{\prime \prime}}}$,

where the theoretical line strengths are normalized according to Whiting \& Nicholls (1974). Note that for transitions between states with $\Lambda \neq 0$, the lines appear as close doublets because of the $\Lambda$-doubling. If such a doublet is not resolved in the observed spectra, the increase of the strength of the blend is to be accounted for by adding the contributions from two lines whose theoretical strengths are identical.

Finally, the radiative damping constant can be expressed as follows:

$\gamma_{\mathrm{R}}=\frac{8 \pi^{2} e^{2}}{m c} \frac{1}{g_{J^{\prime}}} \sum_{\Lambda^{\prime \prime}} \sum_{v^{\prime \prime}} f_{\Lambda v} \lambda_{\Lambda v} \sum_{J^{\prime \prime}} \frac{S_{\Lambda J}}{\lambda_{\Lambda v J}^{3}}$.

We use this formula in the calculations.

\section{Diagnostic capabilities of $C_{2}$}

In the following we apply the theory presented in Sect. 2 to the $\mathrm{C}_{2}$ molecule, and discuss its potential for diagnosing magnetic fields by the Hanle effect.

In the second solar spectrum the $\mathrm{C}_{2}$ lines are the strongest in the $(0,0)$ band of the Swan electronic system $\left(d^{3} \Pi-a^{3} \Pi\right)$ starting from $5165 \AA$ and developing to the blue. In this electronic system lines appear as triplets, which are resolved at low $J$ numbers. For high $J$ numbers the two blue components occur at the same wavelength, while the wavelength difference for the third component reduces. According to Berdyugina et al. (2002) the Landé factor of the middle component is typically more than one order of a magnitude smaller than for the other two components. Therefore, we expect a different sensitivity to the Hanle effect, which is best observed in a resolved triplet.

Using the atlas by Gandorfer (2000) we have searched for prominent and if possible not blended $\mathrm{C}_{2}$ triplets. We have identified the only resolved and unblended triplet $\mathrm{R}_{1}(14)$, $\mathrm{R}_{2}(13), \mathrm{R}_{3}(12)$ at $5140 \AA$. Although there are many unblended but unresolved $\mathrm{P}$ - and R-triplets, we selected the P-triplet at 5141.2 $\AA \mathrm{P}_{1}(42), \mathrm{P}_{2}(41), \mathrm{P}_{3}(40)$, because it is next to the chosen R-triplet. This allows us to combine both triplets in one single observation and avoid many possible sources of errors. Close-by, at $5143.5 \AA$, there is another resolved R-triplet but the two red components are blended with a Fe I line. All calculations are therefore performed for the two chosen triplets.

The selected $\mathrm{C}_{2}$ lines are very weak in the intensity spectrum. Their central depth is smaller than $10 \%$, which implies that the optical thickness of the layer where the $\mathrm{C}_{2}$ lines are formed is smaller than 0.1 , i.e. the lines are optically thin and we can indeed neglect the continuum depolarization. More detailed calculations by Faurobert \& Arnaud (2003) confirm our assumption. For instance, they found that the optical thickness of the layer is about 0.06 .

We employ the solar model atmosphere developed by Grevesse \& Sauval (1999) under the assumption of local thermodynamic equilibrium (LTE). Chemical equilibrium between different atomic and molecular species gives molecular number densities (cf. Berdyugina et al. 2003). We use the solar abundances of C, N, and O recommended by Grevesse et al. (1994). In particular, the $\mathrm{C}$ abundance is $A_{\mathrm{C}}=8.55$, which is important for the $\mathrm{C}_{2}$ molecule.

The linear polarization for the selected lines is obtained according to Eq. (1). In the following we will discuss the different quantities on the right-hand side of Eq. (1) one after the other and finally compare our calculations with the observations from the atlas by Gandorfer (2000).

\subsection{Polarization in the non-magnetic case}

According to Eq. (1) the degree of scattering polarization in an optically thin spectral line and in the absence of a magnetic field depends on the opacity factor, intrinsic polarizability and collisional depolarization factor.

For the opacity factor we have to calculate oscillator strengths and excitation energies and obtain the temperature and number density at the maximum of the line contribution function. We computed the contribution function for the relative depression in the line center in Stokes $I$. Thus, the maximum of the function indicates the depth at which the line effectively forms. A detailed description of the calculated function is given by Grossman-Doerth et al. (1988). The contribution functions for the selected R- and P-triplets at the disk center and the limb angle $\mu=0.1$ corresponding to the observations are shown in Fig. 1. As expected lines at the limb are formed at higher layers. However, it turns out that the P- and R-triplets originate at the same depth for a given angle, even though they have different excitation energies. This allows us to analyze the $\mathrm{P}$ - and R-lines simultaneously because they are formed under similar physical conditions. For example, to compute the opacity factors according to Eq. (2) we use the same temperature $T=5080 \mathrm{~K}$ and the $\mathrm{C}_{2}$ number density $\mathcal{N}_{\max }=0.54 \times 10^{8} \mathrm{~cm}^{-3}$ that correspond to the maximum of the line contribution functions at $\mu=0.1$.

Oscillator strengths are computed employing Eq. (14) where the theoretical line strengths are calculated using formulas from Kovács (1969). Because of the unresolved $\Lambda$-doubling effect, lines strengths are increased by a factor of 2 , which is different e.g. from the numbers given by Kurucz (1993). 
Table 1. $\mathrm{C}_{2}$ line parameters used for the synthesis of the Stokes $Q / I$ profiles.

\begin{tabular}{ccccccccr}
\hline \hline Identification & $\lambda, \AA$ & $g_{J^{\prime \prime}} f_{\Lambda v J}$ & $E_{J^{\prime \prime}}, \mathrm{eV}$ & \multicolumn{1}{c}{$g_{\mathrm{L}}^{\prime}$} & \multicolumn{1}{c}{$W_{2}$} & $K_{\Lambda v J}$ & \multicolumn{1}{c}{$\tau_{\mathrm{R}}, \mathrm{s}$} & $B_{\text {sat }}, \mathrm{G}$ \\
\hline $\mathrm{R}_{1}(14)$ & 5139.926 & 0.742 & 0.2325 & 0.1259 & 0.1300 & $2.356 \cdot 10^{7}$ & $5.746 \times 10^{-8}$ & 40 \\
$\mathrm{R}_{2}(13)$ & 5140.139 & 0.696 & 0.2268 & 0.0100 & 0.1325 & $2.238 \times 10^{7}$ & $5.740 \times 10^{-8}$ & 460 \\
$\mathrm{R}_{3}(12)$ & 5140.376 & 0.653 & 0.2216 & -0.1150 & 0.1353 & $2.125 \times 10^{7}$ & $5.700 \times 10^{-8}$ & 50 \\
\hline $\mathrm{P}_{1}(42)$ & 5141.208 & 2.105 & 0.5513 & 0.0487 & 0.0906 & $3.228 \times 10^{7}$ & $5.719 \times 10^{-8}$ & 125 \\
$\mathrm{P}_{2}(41)$ & 5141.191 & 2.055 & 0.5347 & 0.0017 & 0.0908 & $3.273 \times 10^{7}$ & $5.720 \times 10^{-8}$ & 3500 \\
$\mathrm{P}_{3}(40)$ & 5141.308 & 2.006 & 0.5184 & -0.0485 & 0.0903 & $3.316 \times 10^{7}$ & $5.718 \times 10^{-8}$ & 125 \\
\hline
\end{tabular}

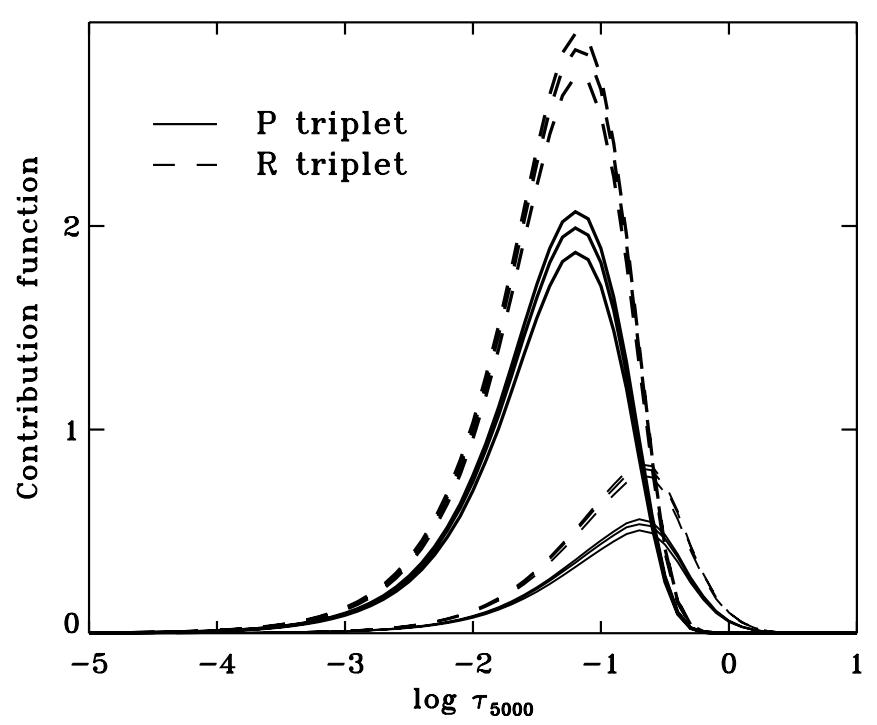

Fig. 1. Contribution functions for the selected $\mathrm{C}_{2} \mathrm{P}$ - and R-triplets at the center of the solar disk (thin lines) and at the limb distance $\mu=0.1$ (thick lines).

The $(0,0)$ band oscillator strength $f_{\Lambda v}$ is taken from Cooper $\&$ Nicholls (1976) and excitation energies are calculated using the molecular constants of Huber \& Herzberg (1979). The resulting opacity factors along with the wavelengths, oscillator strengths and excitation energies for the chosen R- and Ptriplets are given in Table 1. The lines in the P-triplet are a factor of 1.5 stronger than the lines in the R-triplet, mainly because of the oscillator strength which increases with $J$.

The intrinsic polarizability $W_{2}$ is calculated according to Eqs. (3) and (5). For the R-triplet it is about 0.13 and for the P-triplet 0.09 (Table 1). This is in agreement with the general tendency that $\mathrm{R}$ branch lines are always stronger polarized than $\mathrm{P}$ lines for any $J$, as they approach the asymptotic value $W_{2}=$ 0.1 from different sides (Berdyugina et al. 2002).

We neglect a depolarization effect due to elastic collisions for the chosen $\mathrm{C}_{2}$ lines and assume that $W_{\mathrm{c}}=1$. This assumption should be well satisfied because the lines are very weak. Even if $W_{\mathrm{c}}$ slightly differs from 1 its value would be the same for all considered lines so that $W_{\mathrm{c}}$ drops out when studying line ratios in the differential Hanle effect.

\subsection{Hanle depolarization factor $W_{\mathrm{H}}$}

The Hanle depolarization factor $W_{\mathrm{H}}$ given by Eq. (6) involves the calculation of the radiative damping constants and upper level Landé factors. Note that the rate of depolarizing collisions $D^{(2)}=0$ because we neglect elastic collisions here. This means that according to Eq. (7) we effectively overestimate the lifetime of the alignment in the upper level. Therefore, the deduced field strength should be considered as a lower limit.

Radiative damping constants for the selected lines have been calculated with Eq. (15). We neglected the summation over electronic states, because transition probabilities between the upper state $d^{3} \Pi$ and the lower states $c^{3} \Sigma_{u}^{+}$and $b^{3} \Sigma_{g}^{-}$are not known. Therefore, we carried out the summation only for transitions for the lower vibrational states $v^{\prime \prime}=0$ to $v^{\prime \prime}=5$ within the Swan electronic system, using band oscillator strengths from Cooper \& Nicholls (1976).

In order to see how the radiative lifetimes, i.e. the inverse damping constant, depend on the $J$ number, we calculated them for the levels within the $v^{\prime}=0$ vibrational state. These are shown in Fig. 2 compared to the lifetime of the classical oscillator. We found that lifetimes of the $\mathrm{C}_{2}$ levels are on average 5 times longer than that of the classical oscillator. For low $J$ numbers there is a difference in the behavior of the $\mathrm{P}_{1}$ and $\mathrm{P}_{3}$ branches due to the combined effects of the statistical weight and the line strength. According to Eq. (15) the damping constant scales linearly with the ratio of the line strength to the statistical weight. While $g_{J^{\prime}}$ increases monotonically and is the same for both branches, the line strength increases faster for the $\mathrm{P}_{1}$ branch than for the $\mathrm{P}_{3}$. The same effect occurs for the $\mathrm{R}_{1}$ and $\mathrm{R}_{3}$ branches but is less pronounced. The radiative lifetimes for the selected $\mathrm{C}_{2}$ lines are given in Table 1 .

The Landé factors of the upper levels are calculated in the same way as in Berdyugina et al. (2002). Their absolute values are decreasing as $J$ increases. The Landé factors of the sublevels of the $\mathrm{P}_{2}$ and $\mathrm{R}_{2}$ transitions are 10 to 100 times smaller then those of the first and third multiplet sublevels (see Table 1). This influences their sensitivity to the Hanle effect. Signs of the Landé factors of the first and third multiplet sublevels are opposite for $J>4$, which implies opposite sign polarization signals due to the Zeeman effect. This is not distinguished in Stokes $Q / I$ by the Hanle depolarization according to Eqs. (6) and (7) but because of rotation of the polarization plane, we expect to see opposite polarization signatures in Stokes $U / I$ (e.g. Trujillo Bueno 2003c). Observations of such signatures would be a clear confirmation of the Hanle effect in molecular lines.

With the damping constants and the Landé factors we can now compute $W_{\mathrm{H}}$ for a range of magnetic field strengths. Figures 3 and 4 shows $W_{\mathrm{H}}$ for the selected R- and P-triplets, respectively. As expected from Eqs. (6) and (7) $W_{\mathrm{H}}$ falls from 

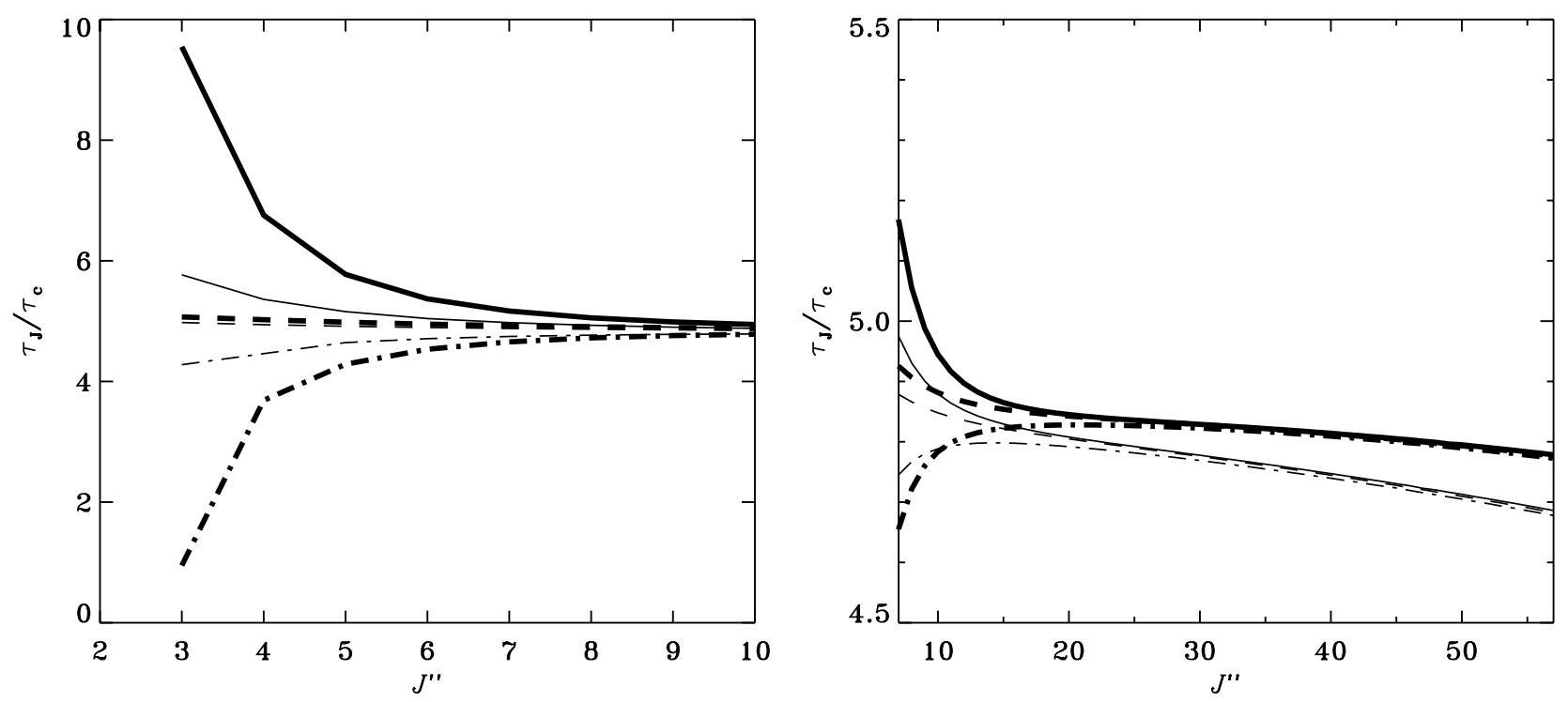

Fig. 2. Radiative lifetimes of the $\mathrm{C}_{2}$ lines from the $(0,0)$ band normalized to the lifetime of the classical oscillator. Thick and thin curves denote lines from the $\mathrm{P}$ and $\mathrm{R}$ branches. The $\mathrm{P}_{1}, \mathrm{P}_{2}, \mathrm{P}_{3}$ as well as $\mathrm{R}_{1}, \mathrm{R}_{2}, \mathrm{R}_{3}$ branches are shown by solid, dashed and dashed-dotted lines, respectively. On the left, the lifetimes for low $J^{\prime \prime}$ numbers are shown and on the right those for higher $J^{\prime \prime}$ numbers.

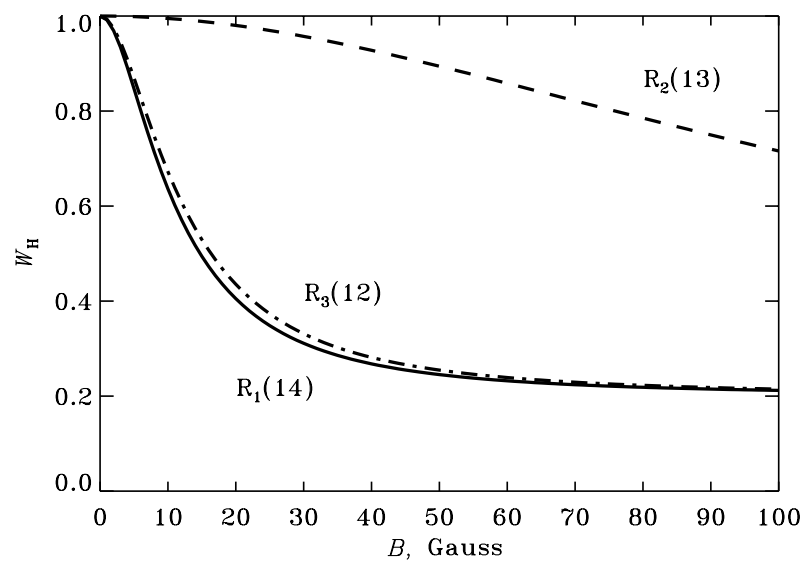

Fig. 3. Hanle depolarizing factor $W_{\mathrm{H}}$ as a function of the magnetic field strength $B$ for the selected R-triplet, which shows the typical behavior of a $\mathrm{P}$ or $\mathrm{R}$ branch triplet of $\mathrm{C}_{2}$ with low $J$ numbers.

the non-magnetic value 1 to the saturation value 0.2 for all lines with non-zero Landé factor. However, the field strength at which the Hanle effect saturates depends on the ratio of the Landé factor to the damping constant, which differs for each line. In both triplets the second branch is less sensitive to the Hanle effect than the first and third branches. This is due to the much smaller Landé factor.

Each line is a useful diagnostic tool only if the Hanle effect is not saturated. Here we call a line saturated at the magnetic field $B_{\text {sat }}$ when $W_{\mathrm{H}} \leq 0.25$. For stronger magnetic fields differences in Hanle depolarization would be barely measurable. The values of $B_{\text {sat }}$ for the two triplets are given in Table 1 . The lines of the first and third branches saturate at field strengths close to $100 \mathrm{G}$ or below, the lines of the second branch at much stronger fields. Because the radiative lifetimes are about the same for all lines, it is the Landé factors that explains the differences in $B_{\text {sat }}$ (see Table 1). For weak magnetic fields up to $20 \mathrm{G}$ the R-triplet provides very good diagnostics since the $\mathrm{R}_{1}(12)$ and

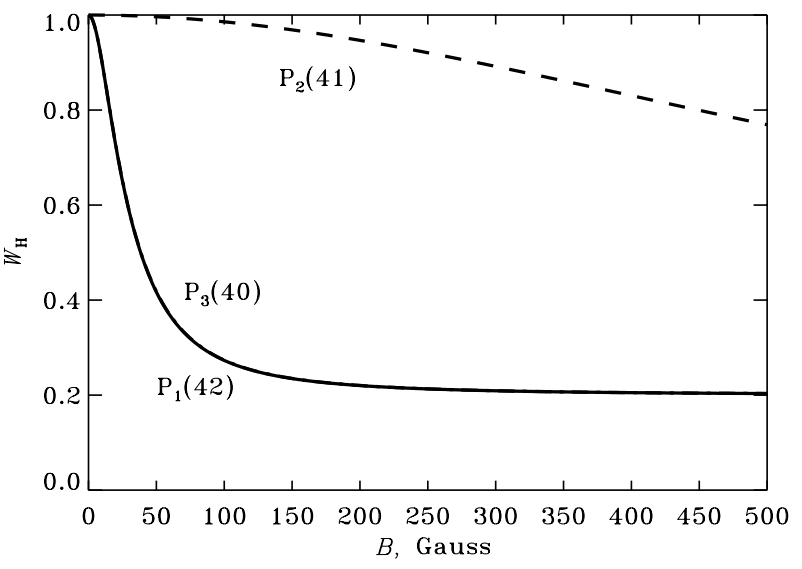

Fig. 4. Hanle depolarizing factor $W_{\mathrm{H}}$ as a function of the magnetic field strength $B$ for the selected P-triplet, which shows the typical behavior of a $\mathrm{P}$ or $\mathrm{R}$ branch triplet of $\mathrm{C}_{2}$ with high $J$ numbers.

$R_{3}(10)$ lines are depolarized quickly while the polarization of the $\mathrm{R}_{2}(11)$ line is not significantly changed and can be used as a reference. The P-triplet behaves analogously but on a magnetic field range up to $100 \mathrm{G}$. At stronger fields, when the first and third branches are saturated, they can be applied as a reference for the polarization scale. Then, the second branch lines provide the means to determine the magnetic field strength up to their value of $B_{\text {sat }}$. However, above $1 \mathrm{kG}$ the Zeeman effect becomes significant and the weak field approximation of the Hanle effect used in this paper is no longer valid.

\subsection{Differential Hanle effect}

The last quantity that remains to be determined before we can diagnose the magnetic field is the scaling factor $q$ in Eq. (1). The different sensitivity to the Hanle effect of the selected lines allows us to obtain $q$. Note that we can well assume the scaling factor to be the same for all chosen lines because they are 

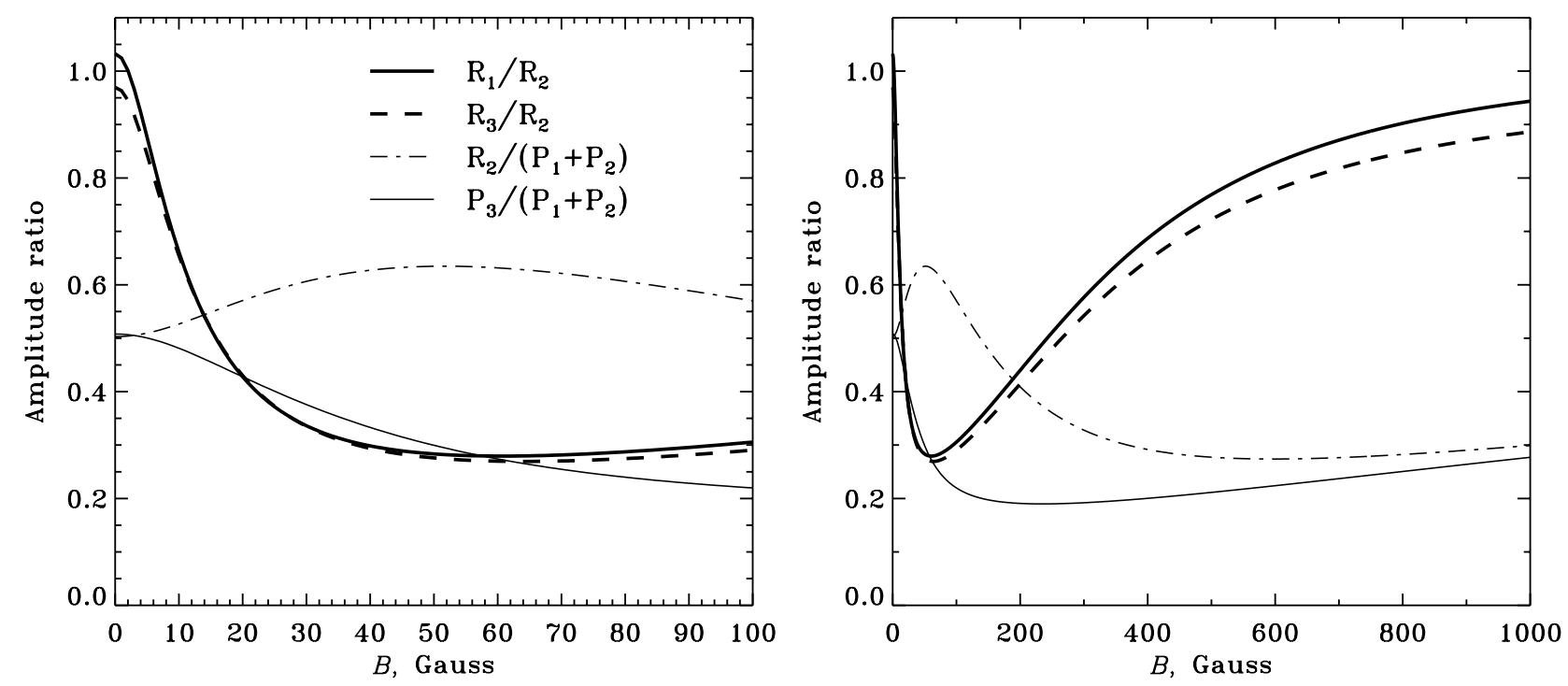

Fig. 5. Polarization amplitude ratios of the $\mathrm{C}_{2}$ lines for two ranges of the magnetic field strength. The line notation is the same for both panels.

formed under very similar physical conditions. In fact, since their formation heights coincide, they are subject to the same magnetic field strength. Therefore, it is possible to employ the differential Hanle effect and study the ratios of the polarization amplitudes of the lines. In this case, the scaling factor $q$ is canceled out.

Figure 5 shows different ratios of the polarization amplitudes of the selected $\mathrm{C}_{2}$ lines. These ratios together allow us to diagnose weak, turbulent magnetic fields over a large range of field strengths from a few Gauss to several hundred Gauss. This is due to the different Hanle effect sensitivities of the considered lines, which is imprinted in the Hanle depolarization factors $W_{\mathrm{H}}$ (cf. Figs. 3 and 4 ).

Take for example the polarization ratio of the $\mathrm{R}_{1}(14)$ to the $\mathrm{R}_{2}$ (13) lines (thick solid line in Fig. 5). In the non-magnetic case the ratio is approximately one, because the lines have comparable strength. With $B$ increasing to $40 \mathrm{G}$, the ratio drops to less than 0.3 because the Hanle effect in the $R_{1}(14)$ line already saturates, while $W_{\mathrm{H}}$ of $\mathrm{R}_{2}(13)$ remains still close to the non-magnetic value of 1 according to Fig. 3. For even greater field strengths the $\mathrm{R}_{2}$ (13) starts to be depolarized also due to the Hanle effect so that the ratio $R_{1} / R_{2}$ approaches again the value 1 , which is reached as soon as the Hanle effect in the $R_{2}(13)$ line saturates as well. Therefore, a given line ratio $R_{1} / R_{2}$ does not correspond to a unique magnetic field strength. Only by taking other ratios into account can the field strength be identified unambiguously because they have a different magnetic field dependence.

Figure 5 represents an excellent diagnostic tool to explore weak magnetic fields. Of particular interest is the R-triplet because the $R_{1}(14)$ and the $R_{3}(12)$ lines are most sensitive to the Hanle effect for magnetic field strengths between a few Gauss to 20 Gauss resulting in the sharp drop of the ratios $R_{1} / R_{2}$ and $R_{3} / R_{2}$. This corresponds exactly to the magnetic field range which has typically been found in earlier studies on the Hanle effect in the second solar spectrum (Faurobert-Scholl 1992, 1993; Bianda et al. 1999). The advantage of the $C_{2}$ lines lies in the fact that the field strength is obtained from the differential Hanle effect and does therefore not depend on the exact knowledge of the degree of scattering polarization in the non-magnetic case.

\subsection{Comparison with observations}

We can now apply our model to the observations presented in the atlas by Gandorfer (2000) and find out whether the Hanle effect is seen in the selected $C_{2}$ lines. The observations in the atlas were acquired between June 1999 and May 2000 using the ZIMPOL II system at the $45 \mathrm{~cm}$ Gregory-Coudé telescope of IRSOL at Locarno, Switzerland. A typical noise level in the $Q / I$ signal is between $10^{-5}$ and $10^{-4}$. The spectra were recorded with the spectrograph slit parallel to the solar limb at a limb distance $\mu=0.1$ near the north pole of the Sun. The total integration time was approximately $30 \mathrm{~min}$ for each measurement and the spectra were averaged along the slit over 180 arcsec of the field of view. Note also that special care was taken to eliminate as much as possible potential modifications of the polarization due to magnetic fields, both by Zeeman and Hanle effects, by simultaneous imaging of the circular polarization. Still, as has been noted by the author, at the time of solar maximum it was not possible to find undisturbed resonance polarization in atomic lines at any place on the solar disk. Therefore, the data in the atlas set a lower limit to the polarization signals that would represent the "non-magnetic" or rather the least magnetic case.

Already a visual check of the R-triplet in the atlas immediately reveals that the polarization amplitudes of the $R_{1}(14)$ and $R_{3}(12)$ lines are modified by the magnetic field, as they are significantly reduced as compared to the amplitude of the $\mathrm{R}_{2}$ (13) line (see Fig. 6). Recall that at zero magnetic field the three lines should be of about the same peak polarization. Thus, at the time of solar maximum even in a magnetically quiet region near the Sun's pole there have been magnetic fields which we can diagnose with the $\mathrm{C}_{2}$ lines. Note, however, that because of severe time and space smearing, we can only speak about a rather average magnetic field. 


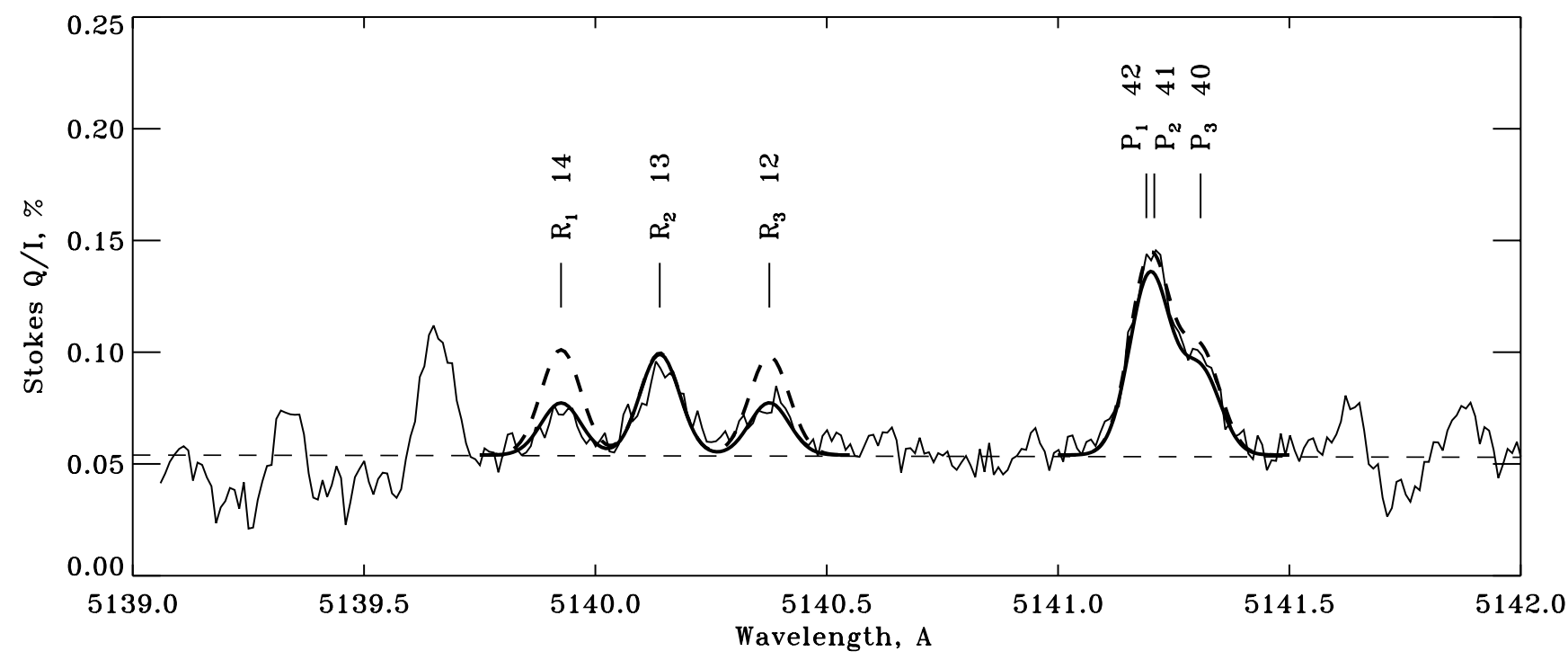

Fig. 6. Fit to the observation of Stokes $Q / I$ in the $\mathrm{C}_{2}$ lines without magnetic field (thick dashed line) and with the magnetic field $B=15 \mathrm{G}$ (thick solid line). The observation from the atlas by Gandorfer (2000) is shown with a thin solid line.

In order to determine the magnetic field strength from the observed Stokes $Q / I$ signals, we synthesized profiles for a range of field strengths using Eq. (1). The damping parameters in the Voigt function were calculated using the radiative damping constants given by Eq. (15), and the Doppler widths of the profiles were adjusted to the observed ones. Since the blue component in the P-triplet is blended with the middle component, we linearly added the two profiles. Such an operation can be justified by the fact that the lines are optically thin. The scaling factor $q=1.54 \times 10^{-8}$ was determined using the $\mathrm{R}_{2}(13)$ line because it remains unaffected by weak magnetic fields.

A simultaneous fit to the R- and P-triplets reveals that the average magnetic field has a strength of $15 \pm 3 \mathrm{G}$. The result is shown in Fig. 6 with the thick solid line. For comparison, the profiles corresponding to the non-magnetic case are shown with the thick dashed line. It is clearly seen that the $\mathrm{R}_{1}(14)$ and $\mathrm{R}_{3}(12)$ lines strongly respond to the weak magnetic field and are excellent diagnostics. At such a magnetic field the P-triplet remains almost undisturbed but, as seen from Fig. 5, it becomes an important diagnostic at fields of $20 \mathrm{G}$ to $70 \mathrm{G}$.

Note that in the previous studies by e.g. Trujillo Bueno (2003c) and Faurobert \& Arnaud (2003) only P-triplets similar to the one selected by us have been analyzed. Our calculations show that for the average field strength below $20 \mathrm{G}$ these lines are rather poor diagnostic tools. This can explain the apparent absence of the magnetic field effect on the scattering polarization of these lines.

\section{Discussion}

We have found the first clear evidence for the Hanle effect in molecular lines, namely in the $\mathrm{C}_{2}$ triplet at $5140 \AA$. The observed magnetic field strength is significantly greater than zero. This eliminates the doubt whether our current model of molecular physics contains a serious shortcoming as compared to atomic physics, and whether molecules behave in principle differently to atoms. Our results are well in agreement with the theory of molecular scattering developed by Berdyugina et al. (2002).

Let us address the issue of apparently different Hanle signatures in molecular and atomic lines. A general overview of the second solar spectrum has led to the impression that molecular lines remain invariant both spatially and in time, whereas many atomic lines are known to vary significantly due to the Hanle effect (Gandorfer 2000; Berdyugina et al. 2002). This does not mean that molecular lines are immune to the Hanle effect, as we have proven in this work. However, there are three main categories of arguments that have to be taken into account in the interpretation of the Hanle effect: (i) the magnetic field range, in which a given line is most sensitive to the Hanle effect; (ii) inhomogeneities of the thermodynamic properties and of the magnetic field distribution in the solar atmosphere; (iii) accuracy and limitations of spectropolarimetric observations. Note that another essential point would be a very basic difference between molecular and atomic physics, which we can rule out as discussed above. In the following we examine in detail these three points.

As pointed out by Trujillo Bueno (2003c) the critical Hanle field in Gauss is given by

$B_{\mathrm{H}} \approx \frac{1.137 \times 10^{-7}}{\tau_{\mathrm{R}} g_{\mathrm{L}}^{\prime}}$

It identifies the approximate magnetic field strength of the highest Hanle effect sensitivity and depends on the product of the radiative lifetime and Landé factor. With the nearly constant lifetime (cf. Fig. 2) and the Landé factor decreasing with $J$, most $\mathrm{C}_{2}$ lines in the studied band have a critical Hanle field greater than $30 \mathrm{G}$. The same is found e.g. for $\mathrm{MgH}$ lines with high $J$ numbers (Trujillo Bueno 2003b). This critical Hanle field has to be compared with the magnetic field strength of $15 \mathrm{G}$ that we have obtained in this work. With such an average turbulent magnetic field strength, it is no surprise that most molecular lines in the second solar spectrum undergo a 
rather weak or even negligible Hanle effect. Note that a magnetic field of typically $10-15 \mathrm{G}$ has also been obtained from observations of the Hanle effect in atomic lines on the quiet Sun (Faurobert-Scholl 1992, 1993; Bianda et al. 1999). But of course, such a comparison has to be regarded with care because it refers to strong atomic lines that form higher in the atmosphere than the $\mathrm{C}_{2}$ lines.

Obviously, molecular lines have to be selected with care to diagnose fields of $10-15 \mathrm{G}$. The $\mathrm{C}_{2}$ R-triplet at $5140 \AA$ that we have identified represents an almost perfect choice for weak field differential Hanle diagnostics. First of all, the three lines have very different critical Hanle fields $B_{\mathrm{H}}$ of about $20 \mathrm{G}$ for the $R_{1}$ and the $R_{3}$ lines, and about $200 \mathrm{G}$ for the $R_{2}$ line. Further, the three lines form under practically identical conditions (same molecule, same excitation energy, same height of formation). With their similar wavelengths the three lines can be observed in a single exposure, thus eliminating many sources of error in observations. And, last but not least, the lines do not blend with each other and the blending by other atomic and molecular lines is negligible.

The second important aspect in the interpretation of the Hanle effect is due to solar physics, namely to the inhomogeneity of the solar atmosphere and the magnetic field distribution. They should lead to spatial variations in the observed Hanle signal. The typical observational signature of the Hanle effect is therefore small scale spatial variations of the degree of scattering polarization in the line core. This signature is regularly recorded in many atomic lines, but usually only in strong lines forming in the upper photosphere or lower chromosphere, such as the Na I D lines or the Ca I $4227 \AA$ line. On the other hand, atomic lines like SrI $4607 \AA$, which is formed rather deep in the photosphere, appear to be spatially rather invariant (Stenflo 2003). Similarly, spatial variations in the scattering polarization of molecular lines (for a given limb distance) have not been found. Therefore, it is not clear whether the claimed difference between atomic and molecular lines is really present.

In addition, all the atomic lines mentioned above belong to the strongest lines in the second solar spectrum with a polarization between $0.3 \%$ and $2 \%$. In comparison, the polarization in molecular lines is of the order of $0.05 \%$ to $0.1 \%$. To obtain the same signal-to-noise ratio, we need to increase the polarimetric sensitivity by at least one order of magnitude for molecular lines. Only then could we conclude whether they show spatial variations. At the moment, the high polarimetric accuracy as in Fig. 6 is only achieved by averaging over the whole spectrograph slit, resulting in a spatial smearing. We plan further observations with a higher polarimetric sensitivity to study the issue of spatial variations in $\mathrm{C}_{2}$.

Trujillo Bueno (2003a,c) has pointed out that the molecular number density should vary spatially due to temperature differences between upflowing and downflowing regions of the photosphere. This is an interesting aspect that could explain potential differences between atomic and molecular lines if they are really significant. However, since the temperature dependence of the chemical equilibrium is different for every molecule, we should not only find dissimilarities between atoms and molecules but also between various molecular species. At the moment, we believe that the most simple and reasonable explanation for the observed spatial variations of Hanle signatures is that inhomogeneities are significant in the upper atmosphere above the temperature minimum, whereas the lower photosphere is much more homogeneous, which explains the lack of spatial variations in both atomic and molecular lines.

The magnetic field distribution, which is present in the solar atmosphere, is also very relevant in Hanle effect studies. In our present model we have assumed an isotropic, turbulent magnetic field of a fixed or averaged strength. Another field distribution would not question the fact that Hanle effect is present in $\mathrm{C}_{2}$ but would correspondingly scale the magnetic field strength for a given Hanle depolarization. Clearly, an isotropic magnetic field is a very simplistic model. Zeeman effect observations show that there is a continuous contribution of magnetic field strengths in the quiet Sun (see e.g. Khomenko et al. 2003). In fact, the magnetic pattern has a large degree of selfsimilarity over all resolved field scales (Stenflo \& Holzreuter 2002, 2003). To extract the real magnetic field distribution on the Sun it is obviously not sufficient to study only a single atom or molecule.

Therefore, let us discuss briefly to what extent our model can be applied to other molecules. We have neglected the effect of continuum depolarization, which is only valid for very weak lines such as $\mathrm{C}_{2}$ or $\mathrm{MgH}$. In stronger lines depolarization should be taken into account (cf. Fluri \& Stenflo 2003). Similarly, effects of optical thickness should be considered in stronger lines such as $\mathrm{CN}, \mathrm{CH}$ or $\mathrm{OH}$ lines. Further, for $\mathrm{C}_{2}$ lines we could safely neglect elastic collisions, which should also be true for lines of the $\mathrm{CN}$ and $\mathrm{MgH}$ molecules. For more abundant molecules like $\mathrm{CH}, \mathrm{OH}$, or $\mathrm{H}_{2}$ scattering polarization might be significantly reduced by elastic collisions. However, the depolarization by elastic collisions drops out in the differential Hanle effect as long as the elastic collision rate is the same in the considered lines. The option to account for elastic collisions is already provided in Eq. (1). Note also that depolarizing elastic collisions reduce the lifetime of the upper level. Thus, when neglecting collisions, the magnetic field strength deduced from the Hanle effect should be considered as a lower limit. Depolarization and optical thickness effects could be included in the model as e.g. proposed by Faurobert \& Arnaud (2003).

In our goal to understand the magnetic field distribution in the solar atmosphere we can only proceed if we study many different lines in both the Hanle and Zeeman regimes. The Zeeman effect is sensitive to the net flux of the magnetic field for field strengths of typically some $100 \mathrm{G}$ and more. The Hanle effect sees also mixed-polarity fields below the resolution limit, but is not present for vertical magnetic fields (at least in the absence of quantum interferences). In addition, observations of different atomic and molecular lines are needed to obtain depthdependent diagnostics. Only by combining Zeeman and Hanle effect observations can we reach a more complete understanding of the solar magnetic fields.

\section{Conclusions}

We developed a simple model for the analysis of scattering polarization of optically thin lines based on the differential Hanle 
effect. Using this model, we analyzed Stokes $Q / I$ profiles of $\mathrm{C}_{2}$ lines observed at the solar limb in a quiet region and showed, for the first time, that molecular lines are significantly depolarized due to the presence of a weak magnetic field.

The optimal choice of lines has aided the progress in our understanding of Hanle effect in molecular lines. The selected $\mathrm{C}_{2}$ R-triplet at $5140 \AA$, being resolved and unblended, satisfies all requirements for employing the differential Hanle effect. It is an excellent diagnostic of magnetic fields weaker than $20 \mathrm{G}$. The P-triplet at $5141 \AA$ A provides complementary constraints for stronger magnetic fields, up to $100 \mathrm{G}$. All the lines form under practically identical conditions in the solar atmosphere (same molecule, same excitation energy, same height of formation), which allows for a simple model of line scattering polarization and reduces potential errors related to assumptions on radiative transfer. Also, having similar wavelengths, the lines can be observed in a single exposure, thus eliminating many sources of errors in observations and instrumental calibrations.

Applying our model to the observation presented in the atlas by Gandorfer (2000), we revealed that the average magnetic field strength in a quiet region near the Sun's north pole at the solar maximum in 1999-2000 was $15 \pm 3 \mathrm{G}$. Since the field strength was obtained using the differential Hanle effect, it does not depend on the exact knowledge of the degree of scattering polarization in the non-magnetic case. However, as the model assumes an isotropic magnetic field distribution, another field distribution would correspondingly scale the magnetic field strength for a given Hanle depolarization. It would not question the fact that Hanle effect is present in the $\mathrm{C}_{2}$ lines.

Note that in the previous studies by e.g. Trujillo Bueno (2003c) and Faurobert \& Arnaud (2003) only P-triplets similar to the one selected by us have been analyzed. Our calculations show that for the average field strength below $20 \mathrm{G}$ these lines are rather poor diagnostic tools. This can explain the apparent absence of the magnetic field effect on the scattering polarization of these lines.

A further confirmation of the Hanle effect in the $C_{2}$ lines would be observations of polarization in Stokes $U / I$. Since the Landé factors of the blue and red components of the R-triplet at $5140 \AA$ are of opposite signs, rotation of the polarization plane due to Hanle effect should result in opposite polarization signatures in Stokes $U / I$.

The fact that spatial variations of scattering polarization have not been yet observed in molecular lines can be due to various reasons, for instance, inadequate choice of lines, relatively low signal-to-noise ratio of observations or a homogeneous magnetic field distribution in the line formation region. Our present results exclude the explanation that molecular lines may not be affected by magnetic fields. We intend, therefore, to observe the selected lines with higher polarimetric sensitivity and put a new limit on the field distribution in the quiet solar atmosphere.

Acknowledgements. We are thankful to Marianne Faurobert and Javier Trujillo Bueno for their useful comments. S.B. acknowledges partial support by the Academy of Finland, grant 43039.

\section{References}

Berdyugina, S. V., Solanki, S. K., \& Frutiger, C. 2003, A\&A, 412, 513 Berdyugina, S. V., Stenflo, J. O., \& Gandorfer, A. 2002, A\&A, 388, 1062

Bianda, M., Stenflo, J. O., \& Solanki, S. K. 1999, A\&A, 350, 1060

Cooper, D. M., \& Nicholls, R. W. 1976, Spectr. Lett., 9, 139

Domke, H., \& Hubeny, I. 1988, ApJ, 334, 527

Faurobert, M., \& Arnaud, J. 2003, A\&A, 412, 555

Faurobert-Scholl, M. 1992, A\&A, 258, 521

Faurobert-Scholl, M. 1993, A\&A, 268, 765

Fluri, D. M., \& Berdyugina, S. V. 2003, in IAU Symp. 219, Stars as Suns: Activity, Evolution, and Planets, ed. A. Benz, \& A. Dupree, in press

Fluri, D. M., \& Stenflo, J. O. 1999, A\&A, 341, 902

Fluri, D. M., \& Stenflo, J. O. 2003, A\&A, 398, 763

Gandorfer, A. 2000, The Second Solar Spectrum, Vol I: $4625 \AA$ to $6995 \AA ̊$ (Zurich: VdF), ISBN No. 3728127647

Grevesse, N., \& Sauval, A. J. 1999, A\&A, 347, 348

Grevesse, N., Sauval, A. J., \& Blomme, R. 1994, in Infrared Solar Physics, ed. D. M. Rabin, J. T. Jefferies, \& C. Lindsey, IAU Symp. 154,539

Grossman-Doerth, U., Larsson, B., \& Solanki, S. 1988, A\&A, 204, 266

Huber, K. P., \& Herzberg, G. 1979, Molecular Spectra and Molecular Structure. IV. Constants of Diatomic Molecules (New York: Van Nostrand Reinhold Company)

Ivanov, V. V. 1991, in Stellar Atmospheres: Beyond Classical Models, NATO ASI Series C 341, ed. L. Crivellari, I. Hubeny, \& D. G. Hummer (Dordrecht: Kluwer), 81

Khomenko, E. V., Collados, M., Solanki, S. K., Lagg, A., \& Trujillo Bueno, J. 2003, A\&A, 408, 1115

Kovács, I. 1969, Rotational Structure in the Spectra of Diatomic Molecules (London: Adam Hilger Ltd.)

Kurucz, R. L. 1993, CD 13

Landi Degl'Innocenti, M., \& Landi Degl'Innocenti, E. 1988, A\&A, 192, 374

Stenflo, J. O. 1982, Sol. Phys., 80, 209

Stenflo, J. O. 1994, Solar Magnetic Fields (Dordrecht: Kluwer)

Stenflo, J. O. 2001, in Advanced Solar Polarimetry - Theory, Observations, and Instrumentation, ed. M. Sigwarth, ASP Conf. Ser., 236, 97

Stenflo, J. O. 2003, in Solar Polarization, ed. J. Trujillo Bueno \& J. Sánchez Almeida, ASP Conf. Ser., 307, 385

Stenflo, J. O., Gandorfer, A., Holzreuter, R., et al. 2002, A\&A, 389, 314

Stenflo, J. O., \& Holzreuter, R. 2002, in Magnetic Coupling of the Solar Atmosphere, ed. H. Sawaya-Lacoste, ESA SP-505 (ESA Publications Division), 101

Stenflo, J. O., \& Holzreuter, R. 2003, in Current Theoretical Models and Future High Resolution Solar Observations: Preparing for ATST, ed. H. Sawaya-Lacoste, ASP Conf. Ser., 286, 169

Stenflo, J. O., Keller, C. U., \& Gandorfer, A. 1998, A\&A, 329, 319

Trujillo Bueno, J. 2001, in Advanced Solar Polarimetry - Theory, Observations, and Instrumentation, ed. M. Sigwarth, ASP Conf. Ser., 236, 161

Trujillo Bueno, J. 2003a, in Modelling of Stellar Atmospheres, ed. N. E. Piskunov, W. W. Weiss, \& D. F. Gray, IAU Symp., 210, 243

Trujillo Bueno, J. 2003b, in Stellar Atmosphere Modeling, ed. I. Hubeny, D. Mihalas, \& K. Werner, ASP Conf. Ser., 288, 551

Trujillo Bueno, J. 2003c, in Solar Polarization, ed. J. Trujillo Bueno \& J. Sánchez Almeida, ASP Conf. Ser., 307, 407

Whiting, E. E., \& Nicholls, R. W. 1974, ApJS, 27, 1 